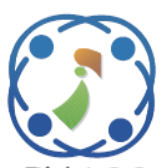

\title{
Fuzzy Observer Design for Fault Estimation for Discrete-time Takagi-Sugeno Singular Models with Unmeasurable Premise Variables
}

\author{
Kaoutar Ouarid ${ }^{1,2 *}$ \\ Abdellatif El Assoudi ${ }^{1,2}$ \\ El Hassane El Yaagoubi ${ }^{1,2}$ \\ Mohamed Essabre ${ }^{3}$ \\ ${ }^{1}$ High Energy Physics and Condensed Matter Laboratory, Faculty of Science, \\ Hassan II University of Casablanca, B.P 5366, Maarif, Casablanca, Morocco \\ ${ }^{2}$ Industrial Process Control Team, Department of Electrical Engineering, ENSEM, \\ Hassan II University of Casablanca, B.P 8118, Oasis, Casablanca, Morocco \\ ${ }^{3}$ Condensed Matter Physics and Renewable Energy Laboratory, \\ Faculty of Sciences and Technologies Mohammedia, Hassan II University of Casablanca, Morocco \\ * Corresponding author's Email: kaoutar.ouarid@ gmail.com
}

\begin{abstract}
This paper aims to present a new approach in the design of observers for a Class of Discrete-time TakagiSugeno Singular Models (DTTSM) in the case of unmeasurable premise variables, allowing to estimate simultaneously the states of the system, and the faults in the actuators and sensors. The approach used is based on augmenting the state vector. The exponential convergence is studied using the Lyapunov theory. The stability conditions are presented as Linear Matrix Inequalities (LMIs). Lastly, a numerical application is used to evaluate the efficiency of the proposed dynamic system. It shows that the estimated variables catch up rapidly (around $0.3 \mathrm{~s}$ ), and accurately the real variables during the application time of the actuator and sensor faults.
\end{abstract}

Keywords: Takagi-sugeno singular model, Discrete-time system, Fuzzy observer, Sensor and actuator faults, Unmeasurable premise variables, LMI, Lyapunov theory.

\section{Introduction}

The advent of microelectronics, control systems and computer technologies has played a major role in the development of industrial processes. Despite the sophistication, evolution and interconnection of equipment, various types of faults are capable of occurring in the process, its control or measuring devices. In order to ensure the continuity of production, and to meet the requirements of reliability, safety, availability, and security, the integration of a fault monitoring and diagnostic system has become essential. This makes information the most important raw material, to know at any time the state of the system and the appearance of abnormal phenomena or even unexpected changes in one or more of its characteristics. Consequently, obtaining this information is based on the presence of a physical sensor or an observer making it possible to collect this information. In this context, the design of an observer has drawn the attention of the scientific community and industrialists in the estimation of states, the control of engineering systems, the detection and diagnosis of faults, as well as faulttolerant control $[1,2]$.

Generally, the study of systems in many practical fields requires going through modeling which represents a crucial phase allowing the representation of the dynamic behavior of the system by a mathematical model. The latter one can be in explicit form described by Ordinary Differential Equations (ODE), or in singular form representing a large class of systems governed by Differential Algebraic Equations (DAE) showing the dynamics and constraints between their variables. Nonetheless, the analysis of nonlinear (NL) singular systems relies on more complex mathematical techniques and tools. Therefore, to facilitate the mathematical exploitation, 
we use the Takagi-Sugeno (T-S) formalism permitting to have a convex polytopic representation based on the extension of the linear results [3, 4]. By using this practical tool, the observer synthesis becomes less complex for the studied system [5].

Many model-based methods [6] have been developed for detection and estimation of states and faults like parity space [7], parametric identification [8], and state estimation [9]. Among the most widespread are those based on observers that have been used with several system classes in the singular or explicit case and in continuous or discrete-time such as: linear parameter varying systems [10], piecewise linear systems [11], linear systems [12], uncertain systems [13], Lipschitz systems [14], and T-S models [2, 15-22].

A wide variety of research has been carried out for the simultaneous estimation of states and faults, at the level of the actuators or the sensors, for the case of T-S fuzzy systems. As an example in the continuous-time, the authors in [16] presented a generalized approach to synthesis a PD observerbased fault estimator for a class of NL systems described by the T-S singular form subject to unknown fault with measurable premise variables. In [2] a new fuzzy functional observer for T-S explicit systems has been considered to estimate the faults affecting the dynamic states of the system. A novel methodology is proposed in [17] to design a robust observer for T-S singular systems considering unmeasurable premise variables and its execution on sensor faults only by considering the robust $\mathrm{H} \infty$ approach. Similarly, in the discrete-time, the authors in [18] designed a robust fault estimation observer for singular systems to estimate actuator faults based on the state augmentation approach. The study in [19] investigated an interval T-S unknown input observer allowing the interval estimation of actuator faults and states for a class of T-S explicit systems.

The aforementioned research papers generally lay focus on the study of the asymptotic convergence of the state and fault estimation errors for a class of T-S systems subject to only one fault at actuator or sensor. The main contribution of our research is to simultaneously estimate the states as well as the faults at the actuator and the sensor for a class of DTSSM. In order to show the convergence of the algebraic part, it proved sufficient to show it just from the convergence of the dynamic part, hence the usefulness of the new approach suggested in this paper. The stability conditions are formulated as linear matrix inequalities. The effectiveness of the proposed approach is demonstrated with the good results obtained during the simulation for a singlelink flexible joint robot, which show from their curves in each figure that the estimated variables by the proposed observer were able to catch up with the variations of real variables.

The organization of this paper will be as follows. In the second section, the class of systems to be studied is described. The third section presents the main purpose of this paper for the simultaneous estimation of states and faults, the stability analysis, and the convergence conditions are demonstrated. Then, the effectiveness of the proposed observer is illustrated by an application example in the fourth section. Finally, the last section is devoted to a summary conclusion.

Throughout this article, these notations are used:

- $\mathrm{B}>0$ means that matrix $\mathrm{B}$ is symmetric and positive definite

- $B^{T}$ is the transpose of matrix B

- The identity and zero matrix are expressed by I and 0 respectively

- The notation $R^{p}$ stands for the p-dimensional Euclidean space and $R^{p \times \mathrm{m}}$ for the set of $\mathrm{p} \times \mathrm{m}$ real matrices

- $\sum_{i, j=1}^{q} \delta_{i} \delta_{j}=\sum_{i=1}^{q} \sum_{j=1}^{q} \delta_{i} \delta_{j}$

\section{Model description}

In this paper, the following class of DTSSM in attendance of actuator and sensor faults is considered:

$$
\left\{\begin{array}{c}
E z_{k+1}=\sum_{i=1}^{q} \mu_{i}\left(\vartheta_{k}\right)\left(A_{i} z_{k}+B_{i} u_{k}+F_{a i} f_{a k}\right) \\
y_{k}=\sum_{i=1}^{q} \mu_{i}\left(\vartheta_{k}\right)\left(C_{i} z_{k}+D_{i} u_{k}+D_{a i} f_{a k}\right. \\
\left.+F_{s i} f_{s k}\right)
\end{array}\right.
$$

where

$Z_{k}=\left[\begin{array}{ll}Z_{k}^{1 T} & Z_{k}^{2 T}\end{array}\right]^{T} \boldsymbol{\epsilon} R^{n}:$ The states vector

$Z_{k}^{1} \epsilon R^{r}$ : The vector of differential variables

$Z_{k}^{2} \epsilon R^{n-r}:$ The vector of algebraic variables

$u_{k} \boldsymbol{\epsilon} R^{m}$ : The control input

$y_{k} \boldsymbol{\epsilon} R^{p}$ : The measured output vector

$f_{a k} \boldsymbol{\epsilon} R^{n_{a}}$ : The vector of actuator faults

$f_{s k} \epsilon R^{n_{s}}$ : The vector of sensor faults

The matrices $E \boldsymbol{\epsilon} R^{n \times \mathrm{n}}$ such that $\operatorname{rank}(\mathrm{E})=\mathrm{r}<\mathrm{n}$, $A_{i} \boldsymbol{\epsilon} R^{n \times \mathrm{n}}, B_{i} \boldsymbol{\epsilon} R^{n \times \mathrm{m}}, F_{a i} \boldsymbol{\epsilon} R^{n \times n_{a}}, C_{i} \boldsymbol{\epsilon} R^{p \times \mathrm{n}}$, $D_{i} \boldsymbol{\epsilon} R^{p \times \mathrm{m}}, D_{a i} \boldsymbol{\epsilon} R^{p \times n_{a}}$, and $F_{s i} \boldsymbol{\epsilon} R^{p \times n_{s}}$ are real known constant matrices : 


$$
\begin{gathered}
E=\left(\begin{array}{ll}
I & 0 \\
0 & 0
\end{array}\right), A_{i}=\left(\begin{array}{ll}
A_{11 i} & A_{12 i} \\
A_{21 i} & A_{22 i}
\end{array}\right) \\
B_{i}=\left(\begin{array}{c}
B_{1 i} \\
B_{2 i}
\end{array}\right), F_{a i}=\left(\begin{array}{c}
F_{a i}^{1} \\
F_{a i}^{2}
\end{array}\right), C_{i}=\left(\begin{array}{ll}
C_{1 i} & C_{2 i}
\end{array}\right)
\end{gathered}
$$

The $A_{22 i}$ are supposed to be invertible and the number of sub-models is represented by q.

The vector containing the premise variable is $\vartheta_{k}$ and the weighting functions are $\mu_{i}\left(\vartheta_{k}\right)$ aiming to ensure the transition between the contributions of each sub model:

$$
\left\{\begin{array}{c}
E z_{k+1}=A_{i} z_{k}+B_{i} u_{k}+F_{a i} f_{a k} \\
y_{k}=C_{i} z_{k}+D_{i} u_{k}+D_{a i} f_{a k}+F_{s i} f_{s k}
\end{array}\right.
$$

Such that the weighting functions check the following properties of the convex sum:

$$
\begin{gathered}
\sum_{i=1}^{q} \mu_{i}\left(\vartheta_{k}\right)=1 ; 0 \leq \mu_{i}\left(\vartheta_{k}\right) \leq 1 ; \\
i=1, \ldots, q
\end{gathered}
$$

Before giving the main result, let us make the following assumption, see [4]:

Assumption 1: Suppose that:

- $\left(E, A_{i}\right)$ are regular, i.e. $\operatorname{det}\left(p E-A_{i}\right) \neq 0$ $\forall p \in C$

- All submodels Eq. (4) are impulse observable and detectable

In order to investigate the fuzzy observer design for DTSSM presented by Eq. (1), the approach is based on the separation between differential and algebraic equations in each sub-model Eq. (4).

By replacing, the matrices of system Eq. (4) by their expressions Eq. (2) and Eq. (3), we obtain the following system:

$$
\left\{\begin{array}{c}
Z_{k+1}^{1}=A_{11 i} Z_{k}^{1}+A_{12 i} Z_{k}^{2}+B_{1 i} u_{k}+F_{a i}^{1} f_{a k} \\
0=A_{21 i} Z_{k}^{1}+A_{22 i} Z_{k}^{2}+B_{2 i} u_{k}+F_{a i}^{2} f_{a k} \\
y_{k}=C_{1 i} Z_{k}^{1}+C_{2 i} Z_{k}^{2}+D_{i} u_{k}+D_{a i} f_{a k} \\
+F_{s i} f_{s k}
\end{array}\right.
$$

From the above form Eq. (6) representing the second equivalent form [4] of system Eq. (4), and taking into account the existence of $A_{22 i}^{-1}$, we obtain:

$$
Z_{k}^{2}=M_{i} Z_{k}^{1}+N_{i} u_{k}+P_{a i} f_{a k}
$$

where

$$
\left\{\begin{array}{l}
M_{i}=-A_{22 i}^{-1} A_{21 i} \\
N_{i}=-A_{22 i}^{-1} B_{2 i} \\
P_{a i}=-A_{22 i}^{-1} F_{a i}^{2}
\end{array}\right.
$$

Substituting the expression Eq. (7) of the algebraic variable $Z_{k}^{2}$ in Eq. (6) gives:

$$
\left\{\begin{array}{c}
Z_{k+1}^{1}=Q_{i} Z_{k}^{1}+R_{i} u_{k}+S_{a i} f_{a k} \\
Z_{k}^{2}=M_{i} Z_{k}^{1}+N_{i} u_{k}+P_{a i} f_{a k} \\
y_{k}=T_{i} Z_{k}^{1}+U_{i} u_{k}+V_{a i} f_{a k}+F_{s i} f_{s k}
\end{array}\right.
$$

where

$$
\left\{\begin{array}{c}
Q_{i}=A_{11 i}+A_{12 i} M_{i} \\
R_{i}=B_{1 i}+A_{12 i} N_{i} \\
S_{a i}=F_{a i}^{1}+A_{12 i} P_{a i} \\
T_{i}=C_{1 i}+C_{2 i} M_{i} \\
U_{i}=D_{i}+C_{2 i} N_{i} \\
V_{a i}=D_{a i}+C_{2 i} P_{a i}
\end{array}\right.
$$

Which is equivalent to the following state representation:

$$
\left\{\begin{array}{c}
Z_{k+1}^{1}=Q_{i} Z_{k}^{1}+R_{i} u_{k}+S_{i} f_{k} \\
Z_{k}^{2}=M_{i} Z_{k}^{1}+N_{i} u_{k}+P_{i} f_{k} \\
y_{k}=T_{i} Z_{k}^{1}+U_{i} u_{k}+V_{i} f_{k}
\end{array}\right.
$$

where

$$
\left\{\begin{array}{c}
f_{k}=\left(\begin{array}{c}
f_{a k} \\
f_{s k}
\end{array}\right) \\
S_{i}=\left(\begin{array}{ll}
S_{a i} & 0
\end{array}\right) \\
P_{i}=\left(\begin{array}{ll}
P_{a i} & 0
\end{array}\right) \\
V_{i}=\left(\begin{array}{ll}
V_{a i} & F_{s i}
\end{array}\right)
\end{array}\right.
$$

Then, from Eq. (11) the weighting functions $\mu_{i}\left(\vartheta_{k}\right)$ can be rewritten as:

$$
\mu_{i}\left(\vartheta_{k}\right)=\mu_{i}\left(Z_{k}^{1}, Z_{k}^{2}=M_{i} Z_{k}^{1}+N_{i} u_{k}+P_{i} f_{k}\right)
$$

$$
=\mu_{i}\left(\varepsilon_{k}\right)
$$

with $\varepsilon_{k}^{T}=\left[\begin{array}{lll}Z_{k}^{1 T} & u_{k}^{T} & f_{k}^{T}\end{array}\right]$

Thus, the global fuzzy model can be represented as: 


$$
\left\{\begin{aligned}
Z_{k+1}^{1} & =\sum_{i=1}^{q} \mu_{i}\left(\varepsilon_{k}\right)\left(Q_{i} Z_{k}^{1}+R_{i} u_{k}+S_{i} f_{k}\right. \\
Z_{k}^{2}= & \sum_{i=1}^{q} \mu_{i}\left(\varepsilon_{k}\right)\left(M_{i} Z_{k}^{1}+N_{i} u_{k}+P_{i} f_{k}\right) \\
y_{k}= & \sum_{i=1}^{q} \mu_{i}\left(\varepsilon_{k}\right)\left(T_{i} Z_{k}^{1}+U_{i} u_{k}+V_{i} f_{k}\right)
\end{aligned}\right.
$$

Assumption 2: Assuming $f_{k}$ as a constant unknown fault signal per time interval i.e.:

$$
\begin{gathered}
f_{k+1}=f_{k} ; \quad k \in\left[T_{1} T_{2}\right] ; \quad \forall T_{1}, T_{2} \\
\in R^{+}
\end{gathered}
$$

In order to simultaneously estimate state and fault vectors, we construct the following augmented state vector such that $X_{k}^{1 T}=\left[\begin{array}{ll}Z_{k}^{1 T} & f_{k}^{T}\end{array}\right]$ and $X_{k}^{2}=$ $Z_{k}^{2}$. Then the equivalent augmented state representation of the system Eq. (13) can be written as follows:

$$
\left\{\begin{aligned}
X_{k+1}^{1} & =\sum_{i=1}^{q} \mu_{i}\left(\omega_{k}\right)\left(\tilde{Q}_{i} X_{k}^{1}+\tilde{R}_{i} u_{k}\right) \\
X_{k}^{2}= & \sum_{i=1}^{q} \mu_{i}\left(\omega_{k}\right)\left(\widetilde{M}_{i} X_{k}^{1}+N_{i} u_{k}\right) \\
y_{k}= & \sum_{i=1}^{q} \mu_{i}\left(\omega_{k}\right)\left(\widetilde{T}_{i} X_{k}^{1}+U_{i} u_{k}\right)
\end{aligned}\right.
$$

where

$$
\left\{\begin{aligned}
\omega_{k} & =\left(\begin{array}{l}
X_{k}^{1} \\
u_{k}
\end{array}\right) \\
\tilde{Q}_{i} & =\left(\begin{array}{cc}
Q_{i} & S_{i} \\
0 & I
\end{array}\right) \\
\tilde{R}_{i} & =\left(\begin{array}{c}
R_{i} \\
0
\end{array}\right) \\
\widetilde{M}_{i} & =\left(\begin{array}{ll}
M_{i} & P_{i}
\end{array}\right) \\
\tilde{T}_{i} & =\left(\begin{array}{ll}
T_{i} & V_{i}
\end{array}\right)
\end{aligned}\right.
$$

\section{Simultaneous state and fault estimation}

In this section, the purpose is to estimate simultaneously the unmeasurable states and unknown faults of DTSSM Eq. (1) or the equivalent system Eq. (15) by proposing a new observer design method.

Thus, the proposed fuzzy observer takes the following form:

$$
\left\{\begin{aligned}
\hat{X}_{k+1}^{1}= & \sum_{i=1}^{q} \mu_{i}\left(\widehat{\omega}_{k}\right)\left(\tilde{Q}_{i} \hat{X}_{k}^{1}+\tilde{R}_{i} u_{k}\right. \\
& \left.-W_{i}\left(\hat{y}_{k}-y_{k}\right)\right) \\
\hat{X}_{k}^{2}= & \sum_{i=1}^{q} \mu_{i}\left(\widehat{\omega}_{k}\right)\left(\widetilde{M}_{i} \hat{X}_{k}^{1}+N_{i} u_{k}\right) \\
\hat{y}_{k}= & \sum_{i=1}^{q} \mu_{i}\left(\widehat{\omega}_{k}\right)\left(\widetilde{T}_{i} \hat{X}_{k}^{1}+U_{i} u_{k}\right)
\end{aligned}\right.
$$

where

$\left(\hat{X}_{k}^{1}, \hat{X}_{k}^{2}\right):$ The estimate of the augmented state vector $\hat{y}_{k}$ : The estimate of the output vector

$\widehat{\omega}_{k}$ : The estimate of the decision variable vector

The gains matrices $W_{i}$ are to be determined such that $\left(\hat{X}_{k}^{1}, \hat{X}_{k}^{2}\right)$ converges to $\left(X_{k}^{1}, X_{k}^{2}\right)$ exponentially.

For establishing the conditions for the asymptotic convergence of the observer Eq. (17), let us define the state estimation error:

$$
\gamma_{k}=\left(\begin{array}{c}
\gamma_{k}^{1} \\
\gamma_{k}^{2}
\end{array}\right)=\left(\begin{array}{l}
\hat{X}_{k}^{1}-X_{k}^{1} \\
\hat{X}_{k}^{2}-X_{k}^{2}
\end{array}\right)
$$

From Eq. (15) and Eq. (17), the dynamics of state estimation error $\gamma_{k}$ are:

$$
\begin{array}{r}
\gamma_{k+1}^{1}=\sum_{i=1}^{q} \mu_{i}\left(\widehat{\omega}_{k}\right)\left(\tilde{Q}_{i} \hat{X}_{k}^{1}+\tilde{R}_{i} u_{k}\right. \\
-W_{i}\left(\hat{y}_{k}\right. \\
\left.\left.-y_{k}\right)\right)-\sum_{\substack{i=1 \\
+}}^{q} \mu_{i}\left(\omega_{k}\right)\left(\tilde{Q}_{i} X_{k}^{1}\right. \\
\left.+\tilde{R}_{i} u_{k}\right) \\
\gamma_{k}^{2}=\sum_{i=1}^{q} \mu_{i}\left(\widehat{\omega}_{k}\right)\left(\widetilde{M}_{i} \hat{X}_{k}^{1}+N_{i} u_{k}\right) \\
-\sum_{i=1}^{q} \boldsymbol{\mu}_{i}\left(\boldsymbol{\omega}_{k}\right)\left(\widetilde{\boldsymbol{M}}_{\boldsymbol{i}} \boldsymbol{X}_{\boldsymbol{k}}^{\mathbf{1}}+\boldsymbol{N}_{\boldsymbol{i}} \boldsymbol{u}_{\boldsymbol{k}}\right)
\end{array}
$$

which are equivalent to the following equations:

$$
\gamma_{k+1}^{1}=\sum_{i=1}^{q} \mu_{i}\left(\widehat{\omega}_{k}\right)\left(\tilde{Q}_{i} \gamma_{k}^{1}-W_{i}\left(\hat{y}_{k}-y_{k}\right)\right)
$$




$$
\begin{aligned}
& -\sum_{i=1}^{q}\left(\mu_{i}\left(\omega_{k}\right)-\mu_{i}\left(\widehat{\omega}_{k}\right)\right)\left(\tilde{Q}_{i} X_{k}^{1}+\tilde{R}_{i} u_{k}\right) \\
& \gamma_{k}^{2}=\sum_{i=1}^{q} \mu_{i}\left(\widehat{\omega}_{k}\right) \widetilde{M}_{i} \gamma_{k}^{1} \\
& -\sum_{i=1}^{q}\left(\mu_{i}\left(\omega_{k}\right)-\mu_{i}\left(\widehat{\omega}_{k}\right)\right)\left(\widetilde{M}_{i} X_{k}^{1}+N_{i} u_{k}\right)
\end{aligned}
$$

From Eq. (21) and Eq. (22), to prove the convergence of the state estimation error $\gamma_{k}$ toward zero, it suffices to prove that $\gamma_{k}^{1}$ converges to zero. Wielding the following writing:

$$
\begin{aligned}
\left(\sum_{i=1}^{q} \mu_{i}\left(\omega_{k}\right)-\right. & \left.\sum_{i=1}^{q} \mu_{i}\left(\widehat{\omega}_{k}\right)\right) K_{i} \\
& =\sum_{i, j=1}^{q} \mu_{i}\left(\omega_{k}\right) \mu_{j}\left(\widehat{\omega}_{k}\right) \Delta K_{i j}
\end{aligned}
$$

With $K_{i}$ can be $\tilde{Q}_{i}, \tilde{R}_{i}, \widetilde{M}_{i}$ or $N_{i}, \Delta K_{i j}=K_{i}-K_{j}$. Then, the differential equation Eq. (21) becomes:

$$
\begin{gathered}
\gamma_{k+1}^{1}=\sum_{i=1}^{q} \mu_{i}\left(\widehat{\omega}_{k}\right)\left(\tilde{Q}_{i} \gamma_{k}^{1}-W_{i}\left(\hat{y}_{k}-y_{k}\right)\right) \\
-\sum_{i, j=1}^{q} \mu_{i}\left(\omega_{k}\right) \mu_{j}\left(\widehat{\omega}_{k}\right)\left(\Delta \widetilde{Q}_{i j} X_{k}^{1}+\Delta \tilde{R}_{i j} u_{k}\right)
\end{gathered}
$$

Since $\sum_{i=1}^{q} \mu_{i}\left(\omega_{k}\right)=1$, the equality Eq. (23) can be written as follows:

$$
\begin{gathered}
\gamma_{k+1}^{1}=\sum_{i, j=1}^{q} \mu_{i}\left(\omega_{k}\right) \mu_{j}\left(\widehat{\omega}_{k}\right)\left(\tilde{Q}_{j} \gamma_{k}^{1}\right. \\
\left.-W_{j}\left(\hat{y}_{k}-y_{k}\right)\right) \\
-\sum_{i, j=1}^{q} \mu_{i}\left(\omega_{k}\right) \mu_{j}\left(\widehat{\omega}_{k}\right)\left(\Delta \tilde{Q}_{i j} X_{k}^{1}+\Delta \tilde{R}_{i j} u_{k}\right)
\end{gathered}
$$

In the same way, the output $y_{k}$ and its estimate can be written as follows:

$$
\begin{gathered}
y_{k}=\sum_{i, h=1}^{q} \mu_{i}\left(\omega_{k}\right) \mu_{h}\left(\widehat{\omega}_{k}\right)\left(\left(\tilde{T}_{h}+\Delta \tilde{T}_{i h}\right) X_{k}^{1}\right. \\
\left.+\left(U_{h}+\Delta U_{i h}\right) u_{k}\right)
\end{gathered}
$$

$\hat{y}_{k}=\sum_{i, h=1}^{q} \mu_{i}\left(\omega_{k}\right) \mu_{h}\left(\widehat{\omega}_{k}\right)\left(\tilde{T}_{h} \hat{X}_{k}^{1}+U_{h} u_{k}\right)$

Such as: $\Delta \widetilde{T}_{i h}=\tilde{T}_{i}-\tilde{T}_{h}$ and $\Delta U_{i h}=U_{i}-U_{h}$

By replacing $y_{k}$ and $\hat{y}_{k}$ with their expressions Eq. (25) and Eq. (26) in Eq. (24), we obtain:

$$
\begin{array}{r}
\gamma_{k+1}^{1} q \\
=\sum_{i, j, h=1} \mu_{i}\left(\omega_{k}\right) \mu_{j}\left(\widehat{\omega}_{k}\right) \mu_{h}\left(\widehat{\omega}_{k}\right)\left(\psi_{j h} \gamma_{k}^{1}\right. \\
\left.+\Theta_{i j h} X_{k}^{1}+\Xi_{i j h} u_{k}\right)
\end{array}
$$

where

$$
\left\{\begin{array}{c}
\psi_{j h}=\tilde{Q}_{j}-W_{j} \widetilde{T}_{h} \\
\Theta_{i j h}=W_{j} \Delta \tilde{T}_{i h}-\Delta \tilde{Q}_{i j} \\
\Xi_{i j h}=W_{j} \Delta U_{i h}-\Delta \tilde{R}_{i j} \\
i, j, h \in(1, \ldots, q)
\end{array}\right.
$$

We set $\tilde{\gamma}_{k}^{1 T}=\left(\gamma_{k}^{1 T} X_{k}^{1 T}\right)$, then we get:

$$
\begin{gathered}
\tilde{\gamma}_{k+1}^{1}=\sum_{i, j, h=1}^{q} \mu_{i}\left(\omega_{k}\right) \mu_{j}\left(\widehat{\omega}_{k}\right) \mu_{h}\left(\widehat{\omega}_{k}\right) \\
\left(\Omega_{i j h} \tilde{\gamma}_{k}^{1}+\Lambda_{i j h} u_{k}\right)
\end{gathered}
$$

where

$$
\left\{\begin{array}{c}
\Omega_{i j h}=\left(\begin{array}{cc}
\psi_{j h} & \Theta_{i j h} \\
0 & \tilde{Q}_{i}
\end{array}\right) \\
\Lambda_{i j h}=\left(\begin{array}{c}
\Xi_{i j h} \\
\tilde{R}_{i}
\end{array}\right)
\end{array}\right.
$$

To present the convergence conditions of the proposed observer Eq. (17), we formulate the following theorem.

Theorem: Under assumptions 1 and 2, the state estimation error between the DTSSM Eq. (1) and its observer Eq. (17) converges exponentially towards zero, if for a scalar $\beta$ between 0 and 1 there exist matrices $Y_{1}>0, Y_{2}>0$ and $L_{i}, i=1, \ldots, q$ such that the following LMIs hold:

$$
\left(\begin{array}{cccc}
\zeta_{11} & \zeta_{21}^{T} & \zeta_{31}^{T} & \zeta_{41}^{T} \\
\zeta_{21} & \zeta_{22} & \zeta_{32}^{T} & \zeta_{42}^{T} \\
\zeta_{31} & \zeta_{32} & \zeta_{33} & \zeta_{43}^{T} \\
\zeta_{41} & \zeta_{42} & \zeta_{43} & \zeta_{44}
\end{array}\right)<0
$$




$$
i, j, h \in(1, \ldots, q)
$$

Where

$$
\left\{\begin{array}{c}
\zeta_{11}=\tilde{Q}_{j}^{T} Y_{1} \tilde{Q}_{j}-\tilde{Q}_{j}^{T} L_{j} \tilde{T}_{h}-\tilde{T}_{h}^{T} L_{j}^{T} \tilde{Q}_{j}-\beta^{2} Y_{1} \\
\zeta_{21}=\Delta \tilde{Q}_{i j}^{T} L_{j} \widetilde{T}_{h}+\Delta \tilde{T}_{i h}^{T} L_{j}^{T} \tilde{Q}_{j}-\Delta \tilde{Q}_{i j} Y_{1} \tilde{Q}_{j} \\
\zeta_{22}=\tilde{Q}_{i}^{T} Y_{2} \tilde{Q}_{i}-\Delta \tilde{Q}_{i j}^{T} L_{j} \Delta \tilde{T}_{i h}+\Delta \tilde{Q}_{i j}^{T} Y_{1} \Delta \tilde{Q}_{i j} \\
\quad-\Delta \tilde{T}_{i h}^{T} L_{j}^{T} \Delta \tilde{Q}_{i j}-\beta^{2} Y_{2} \\
\quad \begin{array}{rl}
\zeta_{31}= & \Delta U_{i h}^{T} L_{j}^{T} \tilde{Q}_{j}^{T}+\Delta \tilde{R}_{i j}^{T} L_{j} \tilde{T}_{h}-\Delta \tilde{R}_{i j}^{T} Y_{1} \tilde{Q}_{j} \\
\zeta_{32}= & \tilde{R}_{i}^{T} Y_{2} \tilde{Q}_{i}+\Delta \tilde{R}_{i j}^{T} Y_{1} \Delta \tilde{Q}_{i j}-\Delta \tilde{R}_{i j}^{T} L_{j} \Delta \tilde{T}_{i h} \\
& \quad-\Delta U_{i h}^{T} L_{j}^{T} \Delta \tilde{Q}_{i j} \\
\zeta_{33}= & \tilde{R}_{i}^{T} Y_{2} \tilde{R}_{i}+\Delta \tilde{R}_{i j}^{T} Y_{1} \Delta \tilde{R}_{i j}-\Delta \tilde{R}_{i j}^{T} L_{j} \Delta U_{i h} \\
\quad-\Delta U_{i h}^{T} L_{j}^{T} \Delta \tilde{R}_{i j}
\end{array} \\
\zeta_{41}=L_{j} \tilde{T}_{h} \quad \\
\zeta_{42}=-L_{j} \Delta \tilde{T}_{i h} \\
\zeta_{43}=-L_{j} \Delta U_{i h} \\
\zeta_{44}=-Y_{1}
\end{array}\right.
$$

As the observer gains in Eq. (17) are determined by:

$$
W_{i}=Y_{1}^{-1} L_{i}
$$

Proof: The theorem is proven by considering the following quadratic Lyapunov function:

$$
V_{k}=\tilde{\gamma}_{k}^{1 T} Y \tilde{\gamma}_{k}^{1}
$$

with

$$
Y=\left(\begin{array}{cc}
Y_{1} & 0 \\
0 & Y_{2}
\end{array}\right)>0
$$

Guaranteeing the following condition ensures the exponential convergence of Eq. (29):

$$
\Delta V_{k}=V_{k+1}-V_{k}<\left(\beta^{2}-1\right) V_{k}
$$

with

$$
0<\beta<1
$$

From Eq. (29) and Eq. (34), the inequality Eq. (36) becomes:

$$
\begin{array}{r}
\Delta V_{k}=\sum_{i, j, h=1}^{q} \mu_{i}\left(\omega_{k}\right) \mu_{j}\left(\widehat{\omega}_{k}\right) \mu_{h}\left(\widehat{\omega}_{k}\right) \\
\left(\tilde{\gamma}_{k}^{1 T} u_{k}^{T}\right) \lambda_{i j h}\left(\begin{array}{c}
\widetilde{\gamma}_{k}^{1} \\
u_{k}
\end{array}\right)<0
\end{array}
$$

$$
\lambda_{i j h}=\left(\begin{array}{cc}
\Omega_{i j h}^{T} Y \Omega_{i j h}-\beta^{2} Y & \Omega_{i j h}^{T} Y \Lambda_{i j h} \\
\Lambda_{i j h}^{T} Y \Omega_{i j h} & \Lambda_{i j h}^{T} Y \Lambda_{i j h}
\end{array}\right)
$$

Substituting $\Omega_{i j h}, \Lambda_{i j h}$, and $Y$ by their expressions Eq. (30) and Eq. (35) in Eq. (38), we obtain:

$$
\begin{gathered}
\lambda_{i j h}=\left(\begin{array}{lll}
\sigma_{11} & \sigma_{21}^{T} & \sigma_{31}^{T} \\
\sigma_{21} & \sigma_{22} & \sigma_{32}^{T} \\
\sigma_{31} & \sigma_{32} & \sigma_{33}
\end{array}\right) \\
i, j, h \in(1, \ldots, q)
\end{gathered}
$$

where

$$
\left\{\begin{array}{l}
\sigma_{11}=\psi_{j h}^{T} Y_{1} \psi_{j h}-\beta^{2} Y_{1} \\
\sigma_{21}=\Theta_{i j h}^{T} Y_{1} \psi_{j h} \\
\sigma_{22}=\Theta_{i j h}^{T} Y_{1} \Theta_{i j h}+\tilde{Q}_{i}^{T} Y_{2} \tilde{Q}_{i}-\beta^{2} Y_{2} \\
\sigma_{31}=\Xi_{i j h}^{T} Y_{1} \psi_{j h} \\
\sigma_{32}=\Xi_{i j h}^{T} Y_{1} \Theta_{i j h}+\tilde{R}_{i}^{T} Y_{2} \tilde{Q}_{i} \\
\sigma_{33}=\Xi_{i j h}^{T} Y_{1} \Xi_{i j h}+\tilde{R}_{i}^{T} Y_{2} \tilde{R}_{i}
\end{array}\right.
$$

The inequality Eq. (37) is satisfied if:

$$
\lambda_{i j h}<0 ; i, j, h \in(1, \ldots, q)
$$

Then, the LMI conditions Eq. (31) defined in the theorem can be established by replacing each matrice by its expression, using the Schur complement and the following change of variables:

$$
L_{j}=Y_{1} W_{j}
$$

Thus, from the Lyapunov stability theory, the error dynamic equation Eq. (29) is exponentially stable if the LMI conditions Eq. (31) are satisfied. This completes the proof of theorem.

\section{Numerical application to single-link flexible Joint Robot}

To test the proposed observer Eq. (17), let us consider the following DTSSM of the single-link flexible joint robot with unmeasurable premise variables that is given by Euler discretisation of the model given in [23] in which actuator and sensor faults have been introduced such that the step size is $\mathrm{T}=0.012 \mathrm{~s}$.

where 


$$
\left\{\begin{array}{c}
E z_{k+1}=\sum_{i=1}^{2} \mu_{i}\left(\vartheta_{k}\right)\left(A_{i} z_{k}+B u_{k}+F_{a} f_{a k}\right) \\
y_{k}=\sum_{i=1}^{2} \mu_{i}\left(\vartheta_{k}\right)\left(C z_{k}+D u_{k}+D a f_{a k}\right. \\
\left.+F_{s} f_{s k}\right)
\end{array}\right.
$$

where

$$
z_{k}=\left[z_{1 k}, \ldots, z_{6 k}\right]: \text { The state vector }
$$

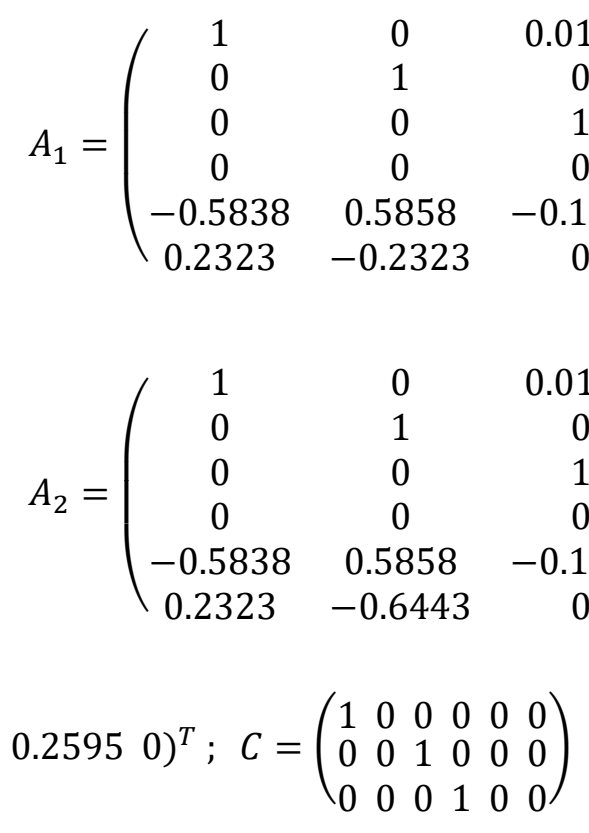

$$
\begin{aligned}
& F_{a}=B \text { and } F_{s}=\left(\begin{array}{lll}
0 & 1 & 0
\end{array}\right)^{T}
\end{aligned}
$$

Under assumption 2, faults $f_{a k}$ and $f_{s k}$ are defined respectively as in Fig. 1 and Fig. 2 which are overlapping during the intervals $[20 \mathrm{~s}, 30 \mathrm{~s}]$ and $[50 \mathrm{~s}$, 60s].

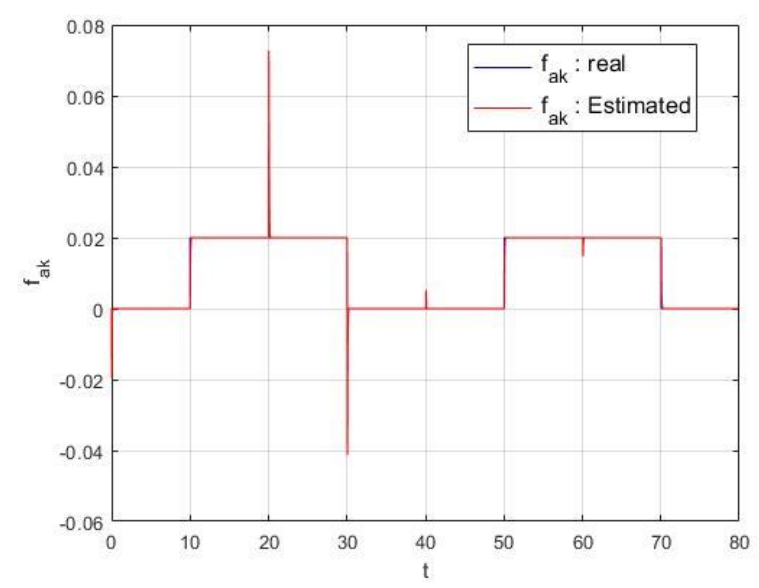

Figure. 1 Actuator fault $f_{a k}$ and its estimate $z_{1 k}:$ The motor rotation angle

$z_{2 k}$ : The link rotation angle

$z_{3 k}$ : The angular velocity of the motor

$z_{4 k}$ : The angular velocity of the link

$z_{5 k}$ and $z_{6 k}$ : Their angular accelerations

$u_{k}$ and $y_{k}$ : The control and the output measurements vectors respectively.

$f_{a k}$ and $f_{s k}$ : The vector of actuator and sensor faults respectively.

The control variable and the physical parameters are given in [23].

$$
\left.\begin{array}{ccc}
0 & 0 & 0 \\
0.0120 & 0 & 0 \\
0 & 0.0120 & 0 \\
1 & 0 & 0.0120 \\
0 & -0.0120 & 0 \\
0 & 0 & -0.0120
\end{array}\right)
$$

$\left.\begin{array}{ccc}0 & 0 & 0 \\ 0.0120 & 0 & 0 \\ 0 & 0.0120 & 0 \\ 1 & 0 & 0.0120 \\ 0 & -0.0120 & 0 \\ 0 & 0 & -0.0120\end{array}\right)$

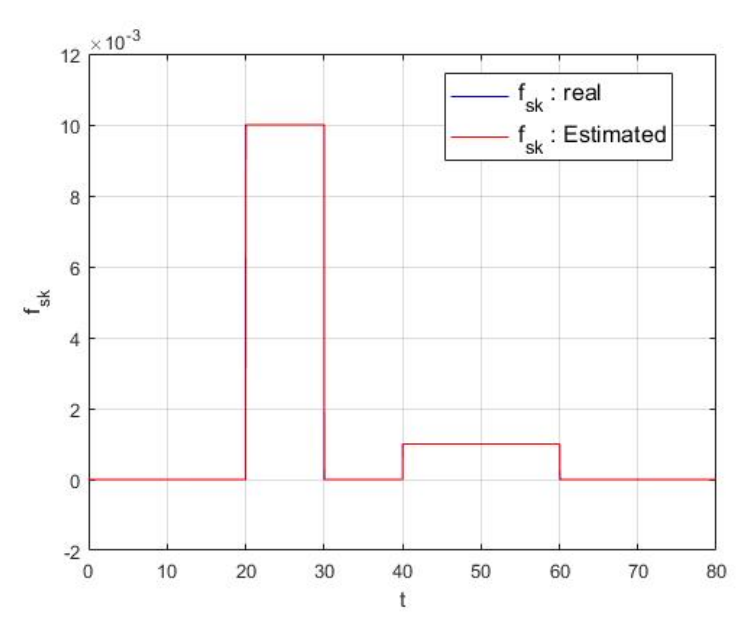

Figure. 2 Sensor fault $f_{s k}$ and its estimate

Therefore, to apply the proposed observer design Eq. (17) for the singular model Eq. (43), as stated in the theorem, it suffices to rewrite the model Eq. (43) into its equivalent form Eq. (15) as previously mentioned. Thus, the resolution of the LMIs defined in the theorem with $\beta=0.5$ leads the following numerical values of the observer gains: 


$$
\begin{gathered}
W_{1}=10^{3}\left(\begin{array}{ccc}
0.0015 & 0 & 0 \\
-0.8102 & 0.0018 & -0.0005 \\
0.0748 & 0.0018 & 0 \\
0.9830 & -0.0009 & 0.0013 \\
1.9670 & -0.0001 & 0.0011 \\
-0.0491 & 0 & 0
\end{array}\right) \\
W_{2}=\left(\begin{array}{ccc}
1.5537 & 0.0120 & 0 \\
207.0810 & 1.4988 & -0.4512 \\
94.5085 & 1.8448 & 0.0010 \\
-27.7870 & -1.1346 & 1.2749 \\
-255.3948 & 0.4606 & 1.0554 \\
-49.4911 & -0.0008 & 0.0005
\end{array}\right)
\end{gathered}
$$

The following simulation results show that the existence of faults leads to an unwanted and remarkable deviation in the system states at the time of the appearance of faults. While, according to the results, we can see, from Fig. 3 to Fig. 8 that the proposed observer designed by using these numerical values of the gain $W_{1}$ and $W_{2}$ allows the estimated states to catch up with real states as quickly as possible, to recover the desired performance, and to ensure the stability despite the presence of faults.

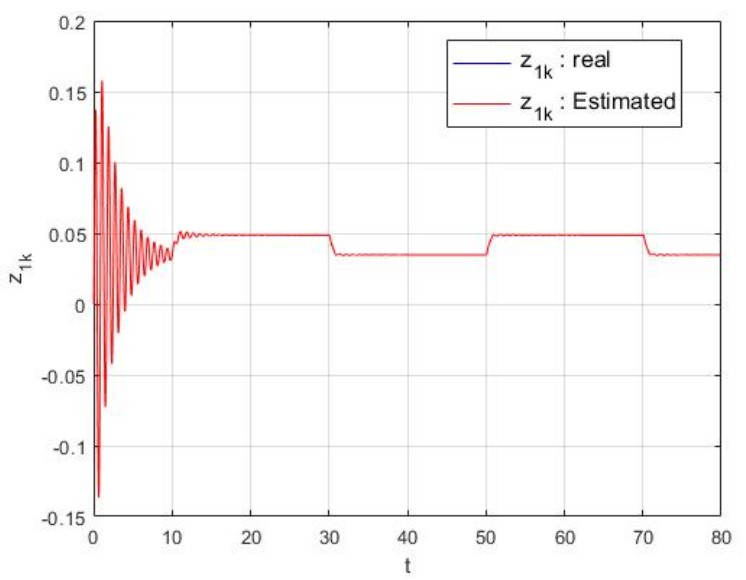

Figure. 3 Motor rotation angle $z_{1 k}$ and its estimate

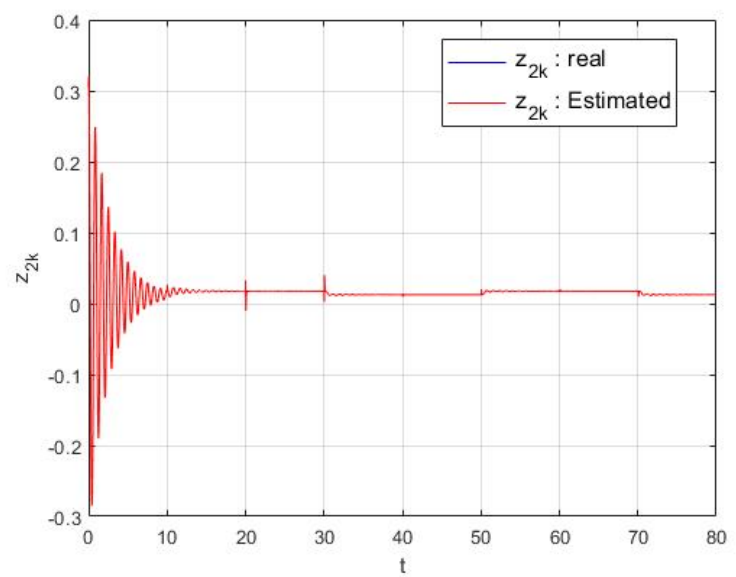

Figure. 4 Link rotation angle $z_{2 k}$ and its estimate

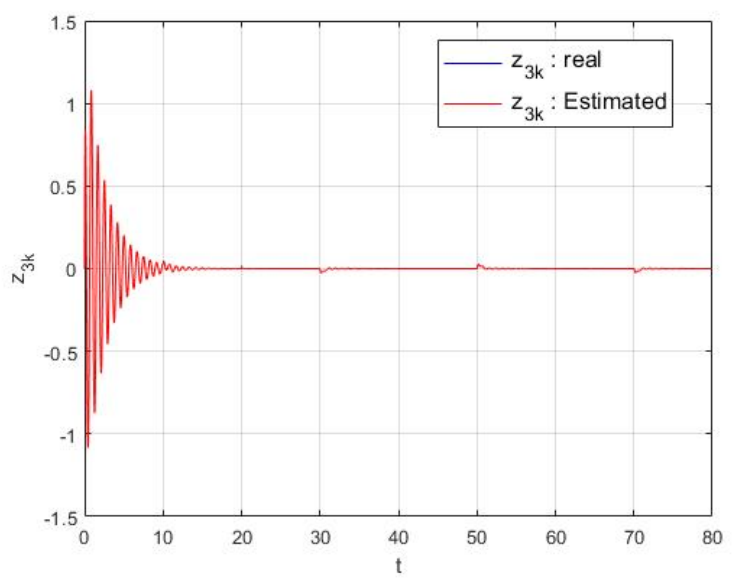

Figure. 5 Angular velocity of the motor $z_{3 k}$ and its estimate

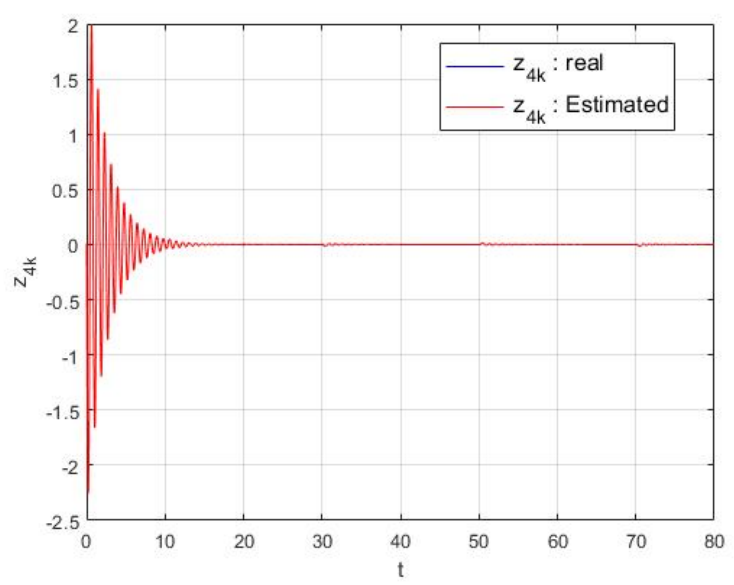

Figure. 6 Angular velocity of the link $z_{4 k}$ and its estimate

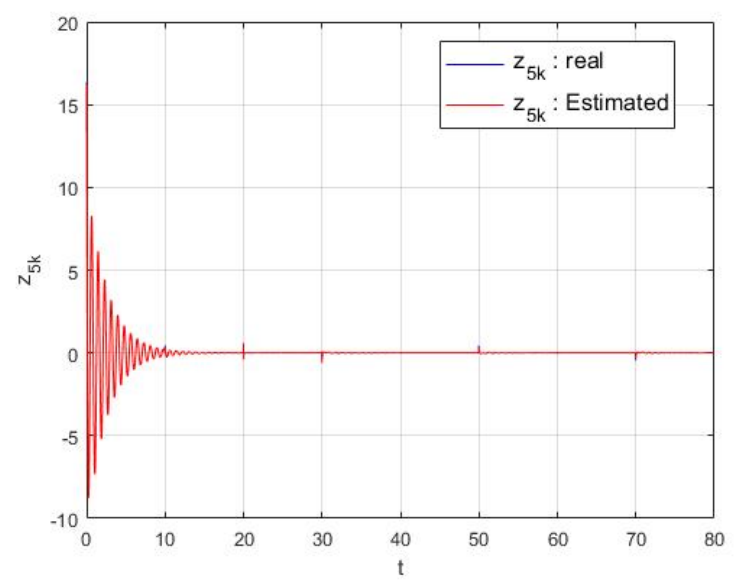

Figure. 7 Angular acceleration of the motor $z_{5 k}$ and its estimate

In $[15,21]$, the simultaneous estimation of states and faults based observer was carried out on the class of T-S models whose premise variables are measured. It is obvious that this class, with premise 


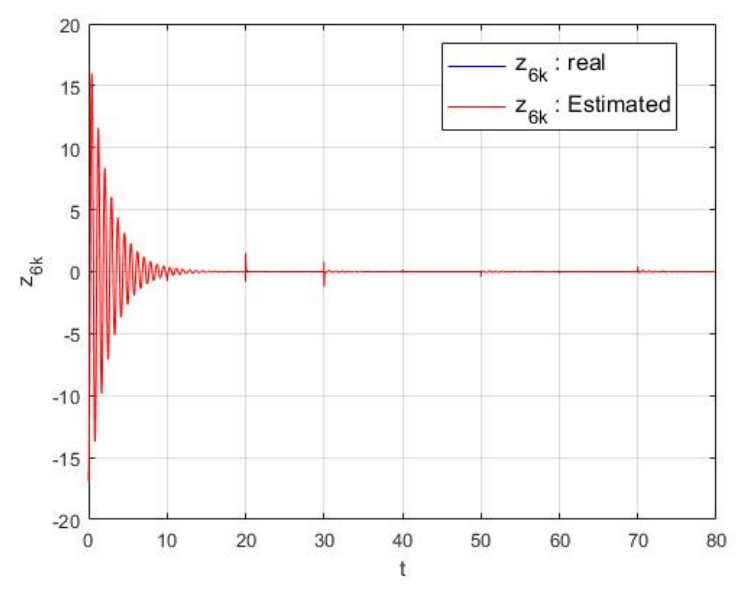

Figure. 8 Angular acceleration of the link $z_{6 k}$ and its estimate

variables relating to the measured variables such as the input or/and the output of the system, is easy to study since the measurements are available. It also serves to benefit from the methods already developed for linear systems, and it will allow a factorization of the weighting functions $\mu_{i}\left(\vartheta_{k}\right)$ when studying the convergence of the estimation error due to the use of the same premise variables on the part of the observer and the model. While in practice, the output considered as a premise variable may be noisy. Therefore, this will have an influence on the accuracy of the model representing the system, as well as the results obtained in this case will not be used for the determination of observer gains in our proposed class. Hence the idea of studying the class of T-S models whose premise variables are unmeasured, representing a large class of NL systems while guaranteeing an exact representation of the NL behaviour of the studied system. Furthermore, the approach presented in [21] is valid in the case of systems guaranteeing the condition of rank mentioned in the paper, while in [22], the resolution of LMIs is related to the value of the Lipschitz constant which must not be larger than an admissible value in order to apply the proposed design method. This motivated us to propose our new approach unrelated to the Lipschitz constant and the rank condition.

\section{Conclusion}

To conclude, a novel observer design method, allowing the contribution to the simultaneous estimation of states and faults, for a class of DTSSM with unmeasurable premise variables is exposed. The proposed result is based on the separation of differential and algebraic equations in the considered model. Thanks to this approach, it turned out that to show the exponential convergence of the state estimation error, it will suffice to study the convergence of the differential part using Lyapunov's theory then conclude the convergence of the algebraic part. The stability conditions are given in terms of LMIs. The usefulness of the proposed observer design for simultaneous estimation of unknown states and faults is well represented by numerical simulations where the proposed observer allows the estimated variables to quickly catch up with the real variables in about $0.3 \mathrm{~s}$.

\section{Conflicts of Interest}

The authors declare no conflict of interest.

\section{Author Contributions}

The paper conceptualization and methodology, Kaoutar Ouarid, Abdellatif El Assoudi and El Hassane El Yaagoubi; software, validation, and formal analysis, Kaoutar Ouarid and Mohamed Essabre; investigation, resources, and data curation, Kaoutar Ouarid and Mohamed Essabre; writingoriginal draft preparation and visualization, Kaoutar Ouarid and Mohamed Essabre; writing-review and editing, Kaoutar Ouarid and Mohamed Essabre; supervision, Abdellatif El Assoudi and El Hassane El Yaagoubi; project administration, Abdellatif El Assoudi and El Hassane El Yaagoubi.

\section{References}

[1] Y. H. Lien, C. C. Peng, and Y. H. Chen, "Adaptive Observer-Based Fault Detection and Fault-Tolerant Control of Quadrotors under Rotor Failure Conditions", Applied Sciences, Vol. 10, No. 10, pp. 1-19, 2020.

[2] S. I. Islam, C. C. Lim, and P. Shi, "Robust fault detection of T-S fuzzy systems with time-delay using fuzzy functional observer", Fuzzy Sets and Systems, Vol. 392, pp.1-23, 2019.

[3] T. Takagi and M. Sugeno, "Fuzzy identification of systems and its application to modeling and control", IEEE Trans. on Systems, Man and Cybernetics, Vol. 15, No. 1, pp. 116-132, 1985.

[4] L. Dai, Singular Control Systems, Vol. 118, Springer, Germany, 1989.

[5] Z. Lendek, T. M. Guerra, R. Babuška, and B. De Schutter, Stability Analysis and Nonlinear Observer Design Using Takagi-Sugeno Fuzzy Models, Vol. 262, Springer, Germany, 2010.

[6] Z. Gao, C. Cecati, and S.X. Ding, "A Survey of Fault Diagnosis and Fault-Tolerant TechniquesPart I: Fault Diagnosis with Model-Based and Signal-Based Approaches", IEEE Trans. on 
Industrial Electronics, Vol. 62, No. 6, pp. 37573767, 2015.

[7] R. J. Patton and J. Chen, "A Review of Parity Space Approaches to Fault Diagnosis", IFAC Proceedings Volumes, Vol. 24, No. 6, pp. 65-81, 1991.

[8] M. Mrugalski and J. Korbicz, "Robust fault diagnosis via parameter identification of dynamical systems", In: Proc. of European Control Conference, Budapest, Hungary, pp. 2923-2928, 2009.

[9] P. M. Frank, "Fault Diagnosis in Dynamic Systems via State Estimation - a Survey", In: Proc. of System Fault Diagnostics, Reliability and Related Knowledge-Based Approaches, Island of Rhodes, Greece, pp. 35-98, 1987.

[10] N. Bedioui, R. Houimli, and M. Besbes, "Simultaneous sensor and actuator fault estimation for continuous-time polytopic LPV system", International Journal of Systems Science, Vol. 50, No. 6, pp. 1290-1302, 2019.

[11] C. Fan, J. Lam, and X. Xie, "Fault detection observer design for periodic piecewise linear systems", International Journal of Systems Science, Vol. 51, No. 9, pp. 1622-1636, 2020.

[12] K. Ouarid, A. E. Assoudi, J. Soulami, and E. E. Yaagoubi, "Observer Design for Simultaneous State and Fault Estimation for a Class of Discrete-time Descriptor Linear Models", In: Proc. of the 3rd International Conference of Computer Science and Renewable Energies, Agadir, Morocco, pp. 1-7, 2021.

[13] J. Zhang, A. Swain, and S. Nguang, "Robust Hळ adaptive descriptor observer design for fault estimation of uncertain nonlinear systems", Journal of the Franklin Institute, Vol. 351, No. 11, pp. 5162-5181, 2014.

[14] Y. Gu, and G. H. Yang, "Simultaneous actuator and sensor fault estimation for discrete-time Lipschitz nonlinear systems in finite-frequency domain", Optimal Control Applications and Methods, Vol. 39, No. 1, pp. 410-423, 2017.

[15] K. Ouarid, A. E. Assoudi, J. Soulami, and E. E. Yaagoubi, "Fault Estimation Based on the Observer Design for Discrete-time TakagiSugeno Descriptor Models", In: Proc. of $3^{\text {rd }}$ International Conference on Intelligent Computing in Data Sciences, Marrakech, Morocco, pp. 1-6, 2019.

[16] D. Krokavec, and A. Filasová, "Design of PD Observer-Based Fault Estimator for a Class of Takagi-Sugeno Descriptor Systems", International Federation of Automatic Control, Vol. 48, No. 11, pp. 754-759, 2015.
[17] F. R. L. Estrada, D. Theilliol, C. M. A. Zaragoza, J. C. Ponsart, G. Valencia-Palomo, and J. L. C. Anzueto, "Fault diagnosis observer for descriptor Takagi-Sugeno systems", Neurocomputing, Vol. 331, pp. 10-17, 2018.

[18] W. Zhang, Z. Wang, T. Raïssi, Y. Wang, and Y. Shen, "A state augmentation approach to interval fault estimation for descriptor systems", European Journal of Control, Vol. 51, pp. 19-29, 2019.

[19] C. Martinez Garcia, V. Puig, C. M. A. Zaragoza, and G.L. Osorio-Gordillo, "Robust Fault Estimation based on Interval Takagi-Sugeno Unknown Input Observer", International Federation of Automatic Control, Vol. 51, No. 24, pp. 508-514, 2018.

[20] K. Ouarid, A. E. Assoudi, J. Soulami, and E. E. Yaagoubi, "Design of Fuzzy Observer for a Class of Takagi-Sugeno Descriptor Models to Simultaneously Estimate States and Faults", Journal of Advanced Research in Dynamical and Control Systems, Vol. 12, No. 5, pp. 239250, 2020.

[21] K. A. Daraou, J. Soulami, A. E. Assoudi, and E. H. E. Yaagoubi, "State and Fault Observer Design for Discrete-time Takagi-Sugeno Descriptor Models", In: Proc. of $3^{\text {rd }}$ International Conference on Intelligent Computing in Data Sciences, Marrakech, Morocco, pp. 1-5, 2019.

[22] M. Ouzaz, A. El Assoudi, J. Soulami, and E. E. Yaagoubi, "Simultaneous state and fault estimation for Takagi-Sugeno implicit models with Lipschitz constraints", International Journal of Optimization and Control: Theories and Applications, Vol. 11, No. 1, pp. 100-108, 2021.

[23] A. Louzimi, A. E. Assoudi, J. Soulami, and E. E. Yaagoubi, "Unknown Input Observer Design for a Class of Nonlinear Descriptor Systems: A Takagi-Sugeno Approach with Lipschitz Constraints", Nonlinear Analysis and Differential Equations, Vol. 5, No. 3, pp. 99-116, 2017. 